\title{
Synthesizing Glue Operators from Glue Constraints for the Construction of Component-Based Systems
}

\author{
Simon Bliudze ${ }^{1}$ and Joseph Sifakis ${ }^{2}$ \\ 1 CEA, LIST, Boîte Courrier 94, Gif-sur-Yvette, F-91191 France \\ Simon.Bliudze@cea.fr \\ 2 VERIMAG, Centre Équation, 2 av de Vignate, 38610, Gières, France \\ Joseph.Sifakis@imag.fr
}

\begin{abstract}
We study glue operators used in component-based frameworks to obtain systems as the composition of atomic components described as labeled transition systems (LTS). Glue operators map tuples of LTS into LTS. They restrict the behavior of their arguments by performing memoryless coordination. In a previous paper, we have proposed a simple format for SOS rules that captures, in particular, glue operators from known frameworks such as CCS, SCCS, CSP, and BIP.

This paper studies a new way for characterizing glue operators: as boolean glue constraints between interactions (sets of ports) and the state of the coordinated components. We provide an SOS format for glue, which allows a natural correspondence between glue operators and glue constraints. This correspondence is used for automated synthesis of glue operators implementing given glue constraints. By focusing on the properties that do not bear computation, we reduce a very hard (and, in general, undecidable) problem of synthesizing controllers to a tractable one. The examples in the paper show that such properties are natural and can be expressed as glue constraints in a straightforward manner. Finally, we compare expressiveness of the proposed formalisms with the glue used in the BIP framework and discuss possible applications.
\end{abstract}

\section{Introduction}

A central idea in systems engineering is that complex systems are built by assembling components. Large components are obtained by "gluing" together simpler ones. "Gluing" can be considered as a generalized composition operation on sets of components that allows building complex components from simpler ones.

Various component frameworks exist for developing hardware, software or mixed hardware/software systems. They all focus rather on the way components interact than on their internal behavior. They often use domain specific mechanisms for composing components, each mechanism implying specific approaches for organizing their interaction. For instance, hardware systems are built by using buses implementing usually synchronous multi-party interaction. 
Asynchronous message passing is very common in operating systems and middleware. For software the main paradigms are using lock/unlock operations or (blocking) function calls, although domain-specific design languages may rely on different mechanisms, e.g., broadcast for synchronous languages like Esterel and rendezvous in ADA. Such a heterogeneity is a main obstacle for

- comparing functionally equivalent designs integrating identical sets of components but using different types of "glue" for their coordination: How to evaluate the merits of different solutions by using theoretical tools and reasoning rather than experimental analysis of their implementations?

- composing systems based on different composition paradigms, often based on different models of computation that cannot be consistently combined.

In previous papers, we have advocated for component frameworks using rather families of composition operators than a single composition operator. This allows mastering complexity in designs and enhanced expressiveness. BIP (BehaviorInteraction-Priority) [1] is such a framework combining two families of composition operators: interactions and priorities. In [2], we formalized the concept of glue operator for behavior coordination by using SOS inference rules in a very simple restriction of the GSOS format [3]. Each operator $g l$ composing behaviors $B_{1}, \ldots, B_{n}$ is defined by a set of inference rules of the form

$$
\frac{\left\{B_{i}: q_{i} \stackrel{a_{i}}{\longrightarrow} q_{i}^{\prime}\right\}_{i \in I} \quad\left\{q_{i}=q_{i}^{\prime}\right\}_{i \notin I} \quad\left\{B_{j}: q_{j} \stackrel{b_{j}^{k}}{\longrightarrow}\right\}_{\substack{j \in J \\ k \in K_{j}}},}{g l\left(B_{1}, \ldots, B_{n}\right): q_{1} \ldots q_{n} \stackrel{a}{\longrightarrow} q_{1}^{\prime} \ldots q_{n}^{\prime}},
$$

where $a$, in the conclusion, is the interaction obtained as the union of the interactions occurring in positive premises.

Example 1 (Mutual exclusion by preemption). Let $T_{1}$ and $T_{2}$ be two mutually preemptable tasks sharing a single processor for their computations. No interactions other than those needed for the preemption mechanism are possible.

Tasks can be modeled by the generic behavior shown to the right. This behavior has three states: 1 -the task is running, 2the task is waiting to begin computation, and 3-the task has been preempted and is waiting to resume computation. The transitions are labeled $b, f, p$, and $r$ for begin, finish, preempt, and resume respectively, and can be synchronized with external

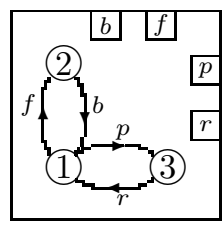
events through the corresponding ports of the behavior. Mutual preemption is described by two statements:

1. A running task is preempted, when the other one begins computation.

2. A preempted task resumes computation, when the other one finishes.

The glue operator $g l$ ensuring this behavior in the composition of the two tasks is defined by the inference rules shown in Fig. 1 (for $i, j=1,2$ and $i \neq j$ ).

In [2], we have introduced a notion of expressiveness for component frameworks and have shown that BIP is as expressive as the family of the glue operators specified by (1). 


$$
\begin{array}{lll}
\frac{B_{i}: q_{i} \stackrel{b_{i}}{\rightarrow} q_{i}^{\prime} \quad B_{j}: q_{j} \stackrel{p_{j}}{\rightarrow}}{g l\left(B_{1}, B_{2}\right): q_{i} q_{j} \stackrel{b_{i}}{\rightarrow} q_{i}^{\prime} q_{j}} & & \frac{B_{i}: q_{i} \stackrel{b_{i}}{\rightarrow} q_{i}^{\prime} \quad B_{j}: q_{j} \stackrel{p_{j}}{\rightarrow} q_{j}^{\prime}}{g l\left(B_{1}, B_{2}\right): q_{i} q_{j} \stackrel{b_{i} p_{j}}{\rightarrow} q_{i}^{\prime} q_{j}^{\prime}} \\
\frac{B_{i}: q_{i} \stackrel{f_{i}}{\rightarrow} q_{i}^{\prime} \quad B_{j}: q_{j} \stackrel{r_{j}}{\rightarrow}}{g l\left(B_{1}, B_{2}\right): q_{i} q_{j} \stackrel{f_{i}}{\rightarrow} q_{i}^{\prime} q_{j}} & & \frac{B_{i}: q_{i} \stackrel{f_{i}}{\rightarrow} q_{i}^{\prime} \quad B_{j}: q_{j} \stackrel{r_{j}}{\rightarrow} q_{j}^{\prime}}{g l\left(B_{1}, B_{2}\right): q_{i} q_{j} \stackrel{f_{i} r_{j}}{\rightarrow} q_{i}^{\prime} q_{j}^{\prime}}
\end{array}
$$

Fig. 1. Inference rules for the mutual exclusion example

This paper studies a new way for characterizing glue operators for behavior coordination. We consider that a glue operator on a set of components is a glue constraint. This is a boolean constraint between interactions that can be fired and the state of the coordinated components. The state is characterized by the set of the ports through which interactions are possible. For each port of a component, the constraint has two variables - an activation variable $p$ and a firing variable $\dot{p}$ - connected by an additional axiom $\dot{p} \Rightarrow p$. For a given valuation of activation variables corresponding to some state of the interacting components, glue constraints characterize all the possible interactions. An interaction is possible from this state if the valuation setting to true only the firing variables corresponding to its ports satisfies the constraint. The axiom $\dot{p} \Rightarrow p$ expresses the fact that a port cannot participate in an interaction unless it is active.

Example 2. The two statements describing preemption in Ex. 1 are formalized by the following constraints (for $i, j=1,2$ and $i \neq j$ ):

$-\dot{p}_{i} \Rightarrow \dot{b_{j}}$, meaning that one task can be preempted only when the other one starts computation;

$-\dot{b_{i}} \wedge p_{j} \Rightarrow \dot{p_{j}}$, meaning that when one task begins computation, if the other one is computing (can be preempted) it must be preempted;

$-\dot{r}_{i} \Rightarrow \dot{f}_{j}$, meaning that a preempted task can resume only when the other one finishes computation;

$-\dot{f}_{i} \wedge r_{j} \Rightarrow \dot{r}_{j}$, meaning that when one task finishes computation, if the other one is preempted (can resume) it must resume.

In this paper, we define a new variation of the SOS format for glue operators proposed in 2]. In the new format, inference rules operators have three types of premises. The firing premises $q_{i} \stackrel{a_{i}}{\rightarrow} q_{i}^{\prime}$ are the same as positive premises in (1). The two other types-witness premises $q_{i} \uparrow a_{i}$ and negative premises $q_{i} \chi a_{i}$-use a new predicate $\uparrow$. In an atomic behavior $B_{i}, q_{i} \uparrow a_{i}$ is satisfied iff, for every port $p \in a_{i}$, there is a transition $q_{i} \stackrel{b}{\rightarrow}$ such that $p \in b$. In other words, every port in $a_{i}$ is offered by some transition in the state $q_{i}$ of $B_{i}$. For a composed behavior $B, q \uparrow a$ signifies that each port in $a$ is offered by some atomic component composing $B$. As in (11), the conclusion of a rule is labeled by the union of interactions labeling its firing premises, that is neither witness nor negative premises contribute to the resulting transition.

This new format has two advantages. Firstly, it is well adapted to formalizing hierarchical composition of behaviors, as the predicate $\uparrow$ allows to explicitly 
capture the notion of "atomic" component. Secondly, for each glue constraint there exists an equivalent glue operator defined by rules in the new SOS format. For any such glue operator, it is possible to find an equivalent glue constraint.

This paper is structured as follows. In Sect. 2, we define a new class of glue operators, which we call universal glue, as well as glue constraints. In Sect. 3 . we show that glue constraints can encode universal glue in a way that allows to explicitly link glue operators to invariant properties of the composed systems. In particular, this allows the synthesis of glue operators enforcing such properties as illustrated in Sect. 4. In Sect. 5, we show that each type of premises in the proposed SOS format is essential for the expressiveness of universal glue. In Sect. 6] we compare this new universal glue with the glue used in BIP. In Sect. 7] we present a design methodology based on the introduced models and transformations. Finally, we discuss the related work in Sect. 8 .

\section{Modeling Behavior and Glue}

\subsection{Behavior}

Definition 1. A labeled transition system (LTS) is a triple $(Q, P, \rightarrow)$, where $Q$ is a set of states, $P$ is a set of ports, and $\rightarrow \subseteq Q \times 2^{P} \times Q$ is a set of transitions, each labeled by an interaction. For $q, q^{\prime} \in Q$ and $a \in 2^{P}$, we write $q \stackrel{a}{\rightarrow} q^{\prime}$ iff $\left(q, a, q^{\prime}\right) \in \rightarrow$. An interaction $a \in 2^{P}$ is active in a state $q \in Q$ (denoted $\left.q \stackrel{a}{\rightarrow}\right)$, iff there exists $q^{\prime} \in Q$ such that $q \stackrel{a}{\rightarrow} q^{\prime}$. We abbreviate $q \stackrel{q}{\rightarrow} \stackrel{\text { def }}{=} \neg(q \stackrel{a}{\rightarrow})$.

To simplify notation, we write, $p q$ for the interaction $\{p, q\}$.

Definition 2. $A$ behavior is a pair $B=(S, \uparrow)$ consisting of an LTS $S=$ $(Q, P, \rightarrow)$ and an offer predicate $\uparrow$ on $Q \times P$ such that $q \uparrow p$ holds (a port $p \in P$ is offered in a state $q \in Q$ ) whenever there is a transition from $q$ containing $p$, that is $\left(\exists a \in 2^{P}: p \in a \wedge q \stackrel{a}{\rightarrow}\right) \Rightarrow q \uparrow p$. The set of ports $P$ is the interface of $B$.

The offer predicate extends to interactions: for $a \in 2^{P}, q \uparrow a \stackrel{\text { def }}{=} \bigwedge_{p \in a} q \uparrow p$. For $p \in P$, we have $q \uparrow p=q \uparrow\{p\}$. It is also important to observe that $\uparrow$ and $\rightarrow$ do not coincide: e.g., for a state $q$ and ports $p_{1}, p_{2}$, if the only transitions possible from $q$ are $q \stackrel{p_{1}}{\rightarrow}$ and $q \stackrel{p_{2}}{\rightarrow}$, one has $q \uparrow p_{1} p_{2}$, but $q \stackrel{p_{f} p_{2}}{\longrightarrow}$.

We write $B=(Q, P, \rightarrow, \uparrow)$ for $B=((Q, P, \rightarrow), \uparrow)$.

Definition 3. A behavior $B=(Q, P, \rightarrow, \uparrow)$ is atomic iff, $\forall q \in Q, p \in P, \quad(q \uparrow$ $\left.p \Leftrightarrow \exists a \in 2^{P}: p \in a \wedge q \stackrel{a}{\rightarrow}\right)$. A behavior is composed if it is not atomic.

According to this definition, the same transition system $S$ can define an atomic or a composed behavior $B=(S, \uparrow)$, depending on the offer predicate. Thus, the offer predicate allows to capture elements of the components in a composed behavior as illustrated by the following example.

Example 3. Consider the following transition systems: 

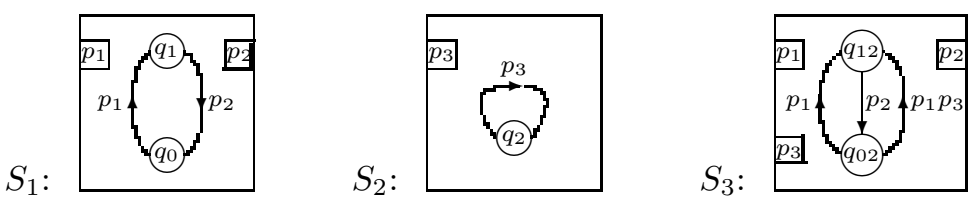

$S_{3}$ can be considered as a composition of $S_{1}$ and $S_{2}$ with an operator that enforces for port $p_{3}$ a synchronization with port $p_{1}$, i.e. each of $p_{1}$ and $p_{2}$ can happen alone, but $p_{3}$ can happen only together with $p_{1}$. Thus, in the state $q_{12}$ of $S_{3}$, there is no transition containing $p_{3}$, even though $p_{3}$ is active in the state 2 of $S_{2}$. This can be reflected by considering the behaviors $B_{i}=\left(S_{i}, \uparrow i\right)$, for $i=1,2,3$, with $\uparrow_{i}$ defined by the first three truth tables below.
$B_{1}:$\begin{tabular}{l|l|l}
$\uparrow_{1}$ & $p_{1}$ & $p_{2}$ \\
\hline$q_{0}$ & $T$ & $F$ \\
\hline$q_{1}$ & $F$ & $T$
\end{tabular}

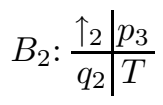
$B_{3}:$\begin{tabular}{l|l|l|l}
$\uparrow_{3}$ & $p_{1}$ & $p_{2}$ & $p_{3}$ \\
\hline$q_{02}$ & $T$ & $F$ & $T$ \\
\hline$q_{12}$ & $F$ & $T$ & $T$
\end{tabular}
$B_{3}^{\prime}:$\begin{tabular}{c|c|c|c}
$\uparrow_{3}^{\prime}$ & $p_{1}$ & $p_{2}$ & $p_{3}$ \\
\hline$q_{02}$ & $T$ & $F$ & $T$ \\
\hline$q_{12}$ & $F$ & $T$ & $\mathbf{F}$
\end{tabular}

Although, there is no interaction $a$ such that $p_{3} \in a$ and, in $B_{3}, q_{12} \stackrel{a}{\rightarrow}$, we have $q_{12} \uparrow p_{3}$, reflecting the fact that, in the behavior $B_{2}$ composing $B_{3}$, the port $p_{3}$ is active. On the other hand, $S_{3}$ can also be considered as "atomic", that is without any explicit information about its structure, by considering a behavior $B_{3}^{\prime}=\left(S_{3}, \uparrow_{3}^{\prime}\right)$ with $\uparrow_{3}^{\prime}$ defined by the fourth truth table above.

Note 1. In the rest of the paper, whenever we speak of a set of component behaviors $B_{i}=\left(Q_{i}, P_{i}, \rightarrow, \uparrow\right)$ with $i \in[1, n]$, we always assume that $\left\{P_{i}\right\}_{i=1}^{n}$ are pairwise disjoint (i.e. $i \neq j$ implies $P_{i} \cap P_{j}=\emptyset$ ) and $P \stackrel{\text { def }}{=} \bigcup_{i=1}^{n} P_{i}$.

Also, to avoid excessive notation, here and in the rest of the paper, we drop the indices on the transition relations $\rightarrow$ and offer predicates $\uparrow$, as they can always be unambigously deduced from the state variables, e.g., $q_{i} \rightarrow$ always refers to the transition relation of the corresponding component $B_{i}$.

\subsection{SOS Characterization of Glue}

Structured Operational Semantics (SOS) 4] has been used to define the meaning of programs in terms of Labeled Transition Systems (LTS). A number of SOS formats have been developed, using various syntactic features [5].

In the context of component-based systems, definition of glue only requires the specification of parallel composition operators, as sequential and recursive computation can be represented by individual behaviors.

The SOS rules format below is a modification of the one defined in [2].

Definition 4. An $n$-ary glue operator $g l$ on a set of interfaces $\left\{P_{i}\right\}_{i=1}^{n}$ is defined as follows. The application of $g l$ to behaviors $B_{i}=\left(Q_{i}, P_{i}, \rightarrow, \uparrow\right)$, for $i \in[1, n]$, is a behavior $\operatorname{gl}\left(B_{1}, \ldots, B_{n}\right)=(Q, P, \rightarrow, \uparrow)$, with

- the set of states $Q=\prod_{i=1}^{n} Q_{i}$-the cartesian product of the sets of states $Q_{i}$,

- the set of ports $P=\bigcup_{i=1}^{n} P_{i}$,

- the minimal offer predicate $\uparrow$ satisfying, for $i \in[1, n]$, the inference rules

$$
\frac{B_{i}: q_{i} \uparrow p}{g l\left(B_{1}, \ldots, B_{n}\right): q_{1} \ldots q_{n} \uparrow p},
$$


- the minimal transition relation $\rightarrow$ satisfying a set of rules of the form

$$
r=\frac{\left\{B_{i}: q_{i} \stackrel{a_{i}}{\longrightarrow} q_{i}^{\prime}\right\}_{i \in I} \quad\left\{B_{j}: q_{j} \uparrow b_{j}\right\}_{j \in J} \quad\left\{B_{k}: q_{k} \not c_{s} \mid s \in L_{k}\right\}_{k \in K}}{g l\left(B_{1}, \ldots, B_{n}\right): q_{1} \ldots q_{n} \stackrel{a}{\longrightarrow} \widetilde{q_{1}} \ldots \widetilde{q_{n}}}
$$

where $I, J, K \subseteq[1, n]$ and $I \neq \emptyset ; a=\bigcup_{i \in I} a_{i}$; and $\widetilde{q}_{i}$ denotes $q_{i}^{\prime}$, for $i \in I$, and $q_{i}$, for $i \in[1, n] \backslash I$. In (3), we have three types of premises respectively called firing, witness, and negative premises. Firing and witness premises are collectively called positive.

The condition $I \neq \emptyset$ means that $r$ has at least one firing premise. Firing premises identify transitions that must actually be taken should the rule be applied; hence there is at most one firing premise per component behavior.

Notice that $q \uparrow b_{1} \wedge q \uparrow b_{2}=q \uparrow b_{1} b_{2}$, i.e. several witness premises can always be merged into a single more complex one. Hence one witness premise per component behavior is sufficient to define any inference rule.

On the contrary, the conjunction of two negative premises $q \not b_{1} \wedge q \not \backslash b_{2}=$ $\neg\left(q \uparrow b_{1} \vee q \uparrow b_{2}\right)$ cannot be expressed as a primitive expression in terms of the transition relation $\rightarrow$ or offer predicate $\uparrow$. Hence, several negative premises for the same component behavior can be necessary to define an inference rule.

A rule is completely defined by its premises.

We identify the glue operator $g l$ with its set of inference rules (3). A glue operator having no negative premises in any of its rules is called a positive glue operator. We call glue a set of glue operators and universal glue the glue consisting of all the glue operators in the sense of Def. 4. We denote the latter $F W N$ (for Firing-Witness-Negative; see also Sect. 5).

\subsection{Boolean Characterization of Glue}

Let $P$ be a set of ports, we denote $\dot{P} \stackrel{\text { def }}{=}\{\dot{p} \mid p \in P\}$.

Definition 5. The Algebra of Glue Constraints on $P$, denoted $\mathcal{G C}(P)$, is the boolean algebra $\mathbb{B}[P, \dot{P}]$ on the set of variables $P \cup \dot{P}$ with an additional axiom $\dot{p} \Rightarrow p$. We call $p \in P$ activation variables and $\dot{p} \in \dot{P}$ firing variables.

Activation variables indicate which ports are offered; firing variables indicate which ports will actually participate in a transition (interaction). Clearly, a port can participate in a transition only if it is offered; hence the axiom $\dot{p} \Rightarrow p$.

Note 2. Below, we overload the symbol $P$ : depending on the context, it will denote the set of ports in a system or the set of the associated boolean variables.

We give the semantics of $\mathcal{G C}(P)$ by associating a glue operator (Def. 4) to each term of the algebra. For behaviors $\left\{B_{i}\right\}_{i=1}^{n}$, such that $P=\bigcup_{i=1}^{n} P_{i}$ where $P_{i}$ is the set of the ports of $B_{i}$, and a glue constraint $\varphi \in \mathcal{G C}(P)$, the composed behavior $\varphi\left(B_{1}, \ldots, B_{n}\right)$ can be described intuitively as follows. For each $i \in[1, n]$, the current state of $q_{i} \in Q_{i}$ defines a valuation on the activation variables of $\varphi$ : 
for each variable $p \in P_{i}$, the valuation is $p=$ true iff $q_{i} \uparrow p$ (the underlying port is offered in the current state of the behavior). An interaction $a \subseteq P$ defines a valuation on the firing variables $\dot{P}$ by putting $\dot{p}=$ true iff $p \in a$. Finally, $a$ is possible in the composite behavior $\varphi\left(B_{1}, \ldots, B_{n}\right)$ iff the valuation on $P \cup \dot{P}$ defined as above satisfies $\varphi$ and the constituent interactions of $a$ are possible in the corresponding component behaviors.

To formalize the above intuition, let us consider behaviors $B_{i}=\left(Q_{i}, P_{i}, \rightarrow, \uparrow\right)$ for $i \in[1, n]$ and a glue constraint $\varphi \in \mathcal{G C}(P)$. Denote $\nu_{B_{i}}^{q_{i}}(p) \stackrel{\text { def }}{=} q_{i} \uparrow p$ the valuation on the activation variables in $P_{i}$ associated to the state $q_{i} \in Q_{i}$ of $B_{i}$. For an interaction $a \subseteq P$, denote $\mathbb{I}_{a}(\dot{p}) \stackrel{\text { def }}{=}(p \in a)$ the corresponding valuation on firing variables. The behavior obtained by composing $\left\{B_{i}\right\}_{i=1}^{n}$ with $\varphi$ is defined by $\varphi\left(B_{1}, \ldots, B_{n}\right)=(Q, P, \rightarrow, \uparrow)$, with $Q=\prod_{i=1}^{n} Q_{i}$, the offer predicate $\uparrow$ defined by (21), and the transition relation $\rightarrow$ defined as follows. For any $q_{1} \ldots q_{n} \in Q$ and $a \subseteq P$, and for all $q_{i}^{\prime} \in Q_{i}$ such that $q_{i} \stackrel{a \cap P_{i}}{\longrightarrow} q_{i}^{\prime}$ for $i \in[1, n]$, we put $q_{1} \ldots q_{n} \stackrel{a}{\rightarrow} q_{1}^{\prime} \ldots q_{n}^{\prime} \stackrel{\text { def }}{\Longleftrightarrow}\left(\nu_{B_{1}}^{q_{1}}, \ldots, \nu_{B_{n}}^{q_{n}}, \mathbb{I}_{a}\right) \models \varphi$.

\section{Transformations}

\subsection{From Glue Operators to Glue Constraints}

Recall that a glue operator on a set of interfaces $\left\{P_{i}\right\}_{i=1}^{n}$ is identified with the set of its defining rules. Thus, we first present the translation into $\mathcal{G C}(P)$ of individual rules. Let $r$ be a rule as in (3). Denoting $A=\bigcup_{i \in I} a_{i}, B=\bigcup_{j \in J} b_{j}$, we associate to $r$ a formula $\varphi_{r} \in \mathcal{G C}(P)$ defined by

$$
\varphi_{r} \stackrel{\text { def }}{=} \bigwedge_{p \in A} \dot{p} \wedge \bigwedge_{p \in P \backslash A} \overline{\dot{p}} \wedge \bigwedge_{p \in B} p \wedge \bigwedge_{k \in K} \bigwedge_{s \in L_{k}} \overline{c_{s}}
$$

where $\overline{c_{s}}=\bigvee_{p \in c_{s}} \bar{p}$. A formula $\varphi_{g l} \in \mathcal{G C}(P)$ associated to a glue operator $g l$ is then defined by putting $\varphi_{g l} \stackrel{\text { def }}{=} \bigvee_{r \in g l} \varphi_{r}$.

Proposition 1. Let $g l \in F W N$ be defined on interfaces $\left\{P_{i}\right\}_{i=1}^{n}$ and let $\varphi_{g l} \in$ $\mathcal{G C}(P)$ be the glue constraint formula constructed as above. For any set of behaviors $B_{i}=\left(Q_{i}, P_{i}, \rightarrow, \uparrow\right)$, with $i \in[1, n]$, holds $g l\left(B_{1}, \ldots, B_{n}\right)=\varphi_{g l}\left(B_{1}, \ldots, B_{n}\right)$.

\subsection{From Glue Constraints to Glue Operators}

Let again $\left\{P_{i}\right\}_{i=1}^{n}$ be a set of interfaces and $P=\bigcup_{i=1}^{n} P_{i}$. The transformation from $\mathcal{G C}(P)$ to $F W N$ can be defined by giving, for a glue constraint formula $\varphi \in \mathcal{G C}(P)$, an equivalent operator $g l_{\varphi} \in F W N$ on $\left\{P_{i}\right\}_{i=1}^{n}$. In order to do so we have to rewrite $\varphi$ as a disjunction of formulæ of the form (44).

Let $\varphi \in \mathcal{G C}(P)$ be a glue constraint formula. Observe first that $\varphi$ has a unique representation in the firing-full $D N F$, i.e. a disjunctive normal form such 
that all firing variables $\dot{p} \in \dot{P}$ are explicitly present in each monomial. Thus $\varphi=\bigvee_{m \in M} \varphi_{m}$, with monomials (indexed by the set $M$ ) of the form

$$
\varphi_{m}=\bigwedge_{p \in A_{m}} \dot{p} \wedge \bigwedge_{p \in P \backslash A_{m}} \overline{\dot{p}} \wedge \bigwedge_{p \in B_{m}} p \wedge \bigwedge_{i=1}^{n} \bigwedge_{p \in C_{m} \cap P_{i}} \bar{p} .
$$

This formula has the same form as in (4). Denote, for $m \in M$ and $i \in[1, n]$, $a_{i}^{m}=A_{m} \cap P_{i}$ and $b_{j}^{m}=B_{m} \cap P_{j} ; I_{m}=\left\{i \in[1, n] \mid a_{i}^{m} \neq \emptyset\right\}$ and $J_{m}=\{j \in$ $\left.[1, n] \mid b_{j}^{m} \neq \emptyset\right\}$. The glue operator $g l_{\varphi}$ corresponding to the formula $\varphi$ is defined by the set of inference rules, containing, for each $m \in M$, the rule ([6]), where $\widetilde{q}_{i}$ denotes $q_{i}^{\prime}$, for $i \in I_{m}$, and $q_{i}$, for $i \in[1, n] \backslash I_{m}$.

$$
\frac{\left\{B_{i}: q_{i} \stackrel{a_{i}^{m}}{\longrightarrow} q_{i}^{\prime}\right\}_{i \in I_{m}} \quad\left\{B_{j}: q_{j} \uparrow b_{j}^{m}\right\}_{j \in J_{m}} \quad\left\{B_{k}: q_{k} \nmid p \mid p \in C_{m} \cap P_{k}\right\}_{k=1}^{n}}{g l_{\varphi}\left(B_{1}, \ldots, B_{n}\right): q_{1} \ldots q_{n} \stackrel{A_{m}}{\longrightarrow} \widetilde{q_{1}} \ldots \widetilde{q_{n}}}
$$

Example 4. Let $P_{1}=\left\{p_{1}, p_{2}\right\}$ and $P_{2}=\left\{p_{3}, p_{4}, p_{5}\right\}$, and consider an operator $g l$ on these two interfaces that allows only transitions labelled $p_{1}$ (i.e. $\dot{p_{1}} \overline{\dot{p_{2}}} \overline{\dot{p_{3}}} \overline{\dot{p_{4}}} \overline{p_{5}}$ ) and, moreover, only when either $p_{2} p_{3}$ is not active or neither is $p_{4}$ nor $p_{5}$ (i.e. $p_{1} \Rightarrow \overline{p_{2} p_{3}} \vee \overline{p_{4}} \overline{p_{5}}$ ). Taking the firing-full DNF of the conjunction, we obtain $\dot{p_{1}} \overline{p_{2}} \overline{p_{3}} \overline{p_{4}} \overline{p_{5}} \wedge\left(\dot{p_{1}} \Rightarrow \overline{p_{2} p_{3}} \vee \overline{p_{4}} \overline{p_{5}}\right)=\dot{p_{1}} \overline{p_{2}} \overline{p_{3}} \overline{p_{4}} \overline{\dot{p_{5}}} \wedge\left(\overline{p_{2} p_{3}} \vee \overline{p_{4}} \overline{p_{5}}\right)=$ $\dot{p_{1}} \overline{\dot{p_{2}}} \overline{p_{3}} \overline{p_{4}} \overline{p_{5}} \overline{p_{2}} \vee \dot{p_{1}} \overline{\dot{p_{2}}} \overline{p_{3}} \overline{p_{4}} \overline{\dot{p_{5}}} \overline{p_{3}} \vee \dot{p_{1}} \overline{\dot{p_{2}}} \overline{\dot{p_{3}}} \overline{\dot{p_{4}}} \overline{\dot{p_{5}}} \overline{p_{4}} \overline{p_{5}}$. The inference rules for the glue operator $g l$ are shown below:

$$
\begin{gathered}
\frac{B_{1}: q_{1} \stackrel{p_{1}}{\rightarrow} q_{1}^{\prime} \quad B_{1}: q_{1} \rtimes p_{2}}{g l\left(B_{1}, B_{2}\right): q_{1} q_{2} \stackrel{p_{1}}{\longrightarrow} q_{1}^{\prime} q_{2}} \quad \frac{B_{1}: q_{1} \stackrel{p_{1}}{\longrightarrow} q_{1}^{\prime} \quad B_{1}: q_{1} \rtimes p_{3}}{g l\left(B_{1}, B_{2}\right): q_{1} q_{2} \stackrel{p_{1}}{\longrightarrow} q_{1}^{\prime} q_{2}} \\
\frac{B_{1}: q_{1} \stackrel{p_{1}}{\longrightarrow} q_{1}^{\prime} \quad B_{2}: q_{2} \rtimes p_{4} \quad B_{2}: q_{2} \rtimes p_{5}}{g l\left(B_{1}, B_{2}\right): q_{1} q_{2} \stackrel{p_{1}}{\longrightarrow} q_{1}^{\prime} q_{2}}
\end{gathered}
$$

Lemma 1. Let $\varphi \in \mathcal{G C}(P)$ be a glue constraint, gl $l_{\varphi} \in F W N$ constructed as above, and $\varphi_{g l_{\varphi}} \in \mathcal{G C}(P)$ constructed as in Sect. 3.1 to $g l_{\varphi}$. Then $\varphi_{g l_{\varphi}}=\varphi$.

Proposition 2. Let $\left\{P_{i}\right\}_{i=1}^{n}$ be a set of interfaces, $\varphi \in \mathcal{G C}(P)$ a constraint formula, and $\mathrm{gl}_{\varphi} \in F W N$ constructed as above. For any set of behaviors $B_{i}=$ $\left(Q_{i}, P_{i}, \rightarrow, \uparrow\right)$, with $i \in[1, n]$, we have $\varphi\left(B_{1}, \ldots, B_{n}\right)=g l_{\varphi}\left(B_{1}, \ldots, B_{n}\right)$.

\section{Synthesis of Glue: A Rescue Robot Example}

The following example was inspired by the hill-climbing robot discussed in [6]. Consider a robot $R$ confined to a square paved region (Fig. 2( $(a)$ ) and consisting of four modules: an engine $E$, a sensor $S$, a transmitter $T$, and a navigation system $N$. The engine can perform two actions: advance the robot one step forward and rotate $90^{\circ}$, controlled respectively through ports a (advance) and $r$ (rotate). The sensor can measure the temperature in front of the robot and signal whether it is above a given threshold. The action of measuring is controlled through the port $m$ (measure), whereas the produced signal can be observed on the port $h(h o t)$. 
(a)

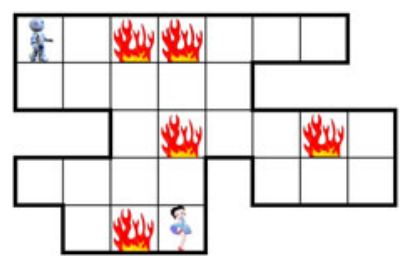

(b)

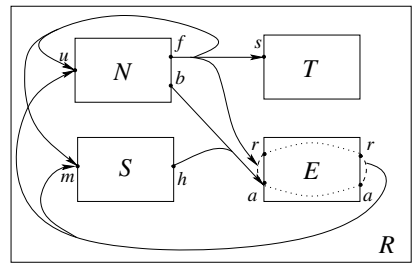

Fig. 2. (a) A rescue robot confined to a square paved region saving Betty Boop from fire; $(b)$ architectural view of the robot.

The transmitter can send the robot's coordinates to the mission control. This action is controlled through the port $s$ (send). Finally, the navigation system detects whether the robot is facing the border of the region and whether it has reached the objective. The navigation system data can be updated by an action controlled through the port $u$ (update), the proximity of the border is signalled through the port $b$ (border) and the objective through the port $f$ (finish). Thus, the four interfaces are $P_{E}=\{a, r\}, P_{S}=\{m, h\}, P_{T}=\{s\}$, and $P_{N}=\{u, b, f\}$.

We want to synthesize the glue ensuring the following properties, which we encode by glue constraints. Notice, that the synthesized glue only has to ensure the safety of the robot and not that it reaches an objective.

1. The robot must not advance and rotate at the same time: $\overline{\dot{a}} \dot{r}$;

2. The robot must not leave the region: $b \Rightarrow \overline{\dot{a}}$;

3. The robot must not drive into hot areas: $h \Rightarrow \overline{\dot{a}}$;

4. If the robot reaches the objective, it must stop and transmit its coordinates: $f \Rightarrow \overline{\dot{a}} \overline{\dot{r}} \overline{\dot{u}} \overline{\dot{m}} \dot{s}$

5. The robot must only transmit its coordinates when it reaches the objective: $\dot{s} \Rightarrow f$;

6. Every time the robot moves (advances or rotates) the navigation system and the sensor must update their data: $\dot{a} \vee \dot{r} \Rightarrow \dot{u} \dot{m}$.

The architecture of the robot is illustrated in Fig. 2(b), with the controllable ports shown on the left-hand side of each component and the controlling ones on the right-hand side. The ports $a$ and $r$ of the engine are both controllable and controlling, so we duplicate them. The arrows indicate the control influence given by the constraints above.

In order to compute the required glue, we take the conjunction of the six constraints above and the progress constraint $(\dot{a} \vee \dot{r} \vee \dot{u} \vee \dot{m} \vee \dot{s}) \wedge \overline{\dot{h}} \bar{b} \bar{f}$-stating that some controllable action $(a, r, u, m$, or $s)$ must be taken, but not the noncontrollable actions $h, b$, and $f$-and apply the procedure described in Sect. 3.2 We rewrite the obtained glue constraint formula in the firing-full DNF: $\overline{\dot{a}} \dot{r} \wedge(b \Rightarrow$ $\overline{\dot{a}}) \wedge(h \Rightarrow \overline{\dot{a}}) \wedge(f \Rightarrow \overline{\dot{a}} \overline{\dot{r}} \overline{\dot{u}} \bar{m} \dot{s}) \wedge(\dot{s} \Rightarrow f) \wedge(\dot{a} \vee \dot{r} \Rightarrow \dot{u} \dot{m}) \wedge(\dot{a} \vee \dot{r} \vee \dot{u} \vee \dot{m} \vee \dot{s}) \wedge \overline{\dot{h}} \dot{b} \overline{\dot{f}}=$

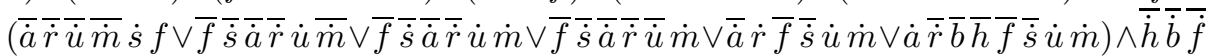
Positive parts of all the monomials in this formula are distinct. Therefore no further transformations are necessary, and we directly obtain the following rules 
defining the required glue operator (we drop the component names, as they are clear from the context):

$$
\begin{gathered}
\frac{q_{n} \stackrel{u}{\rightarrow} q_{n}^{\prime} \quad q_{n} \not \supset f}{q_{e} q_{s} q_{n} q_{t} \stackrel{u}{\rightarrow} q_{e} q_{s} q_{n}^{\prime} q_{t}}, \frac{q_{s} \stackrel{m}{\rightarrow} q_{s}^{\prime} q_{n} \stackrel{u}{\rightarrow} q_{n}^{\prime} q_{n} \not f f}{q_{e} q_{s} q_{n} q_{t} \stackrel{m u}{\longrightarrow} q_{e} q_{s}^{\prime} q_{n}^{\prime} q_{t}}, \frac{q_{s} \stackrel{m}{\rightarrow} q_{s}^{\prime}}{q_{e} q_{s} q_{n} q_{t} \stackrel{m}{\rightarrow} q_{e} q_{s}^{\prime} q_{n} q_{t}}, \\
\frac{q_{t} \stackrel{s}{\rightarrow} q_{t}^{\prime} \quad q_{n} \uparrow f}{q_{e} q_{s} q_{n} q_{t} \stackrel{s}{\rightarrow} q_{e} q_{s} q_{n} q_{t}^{\prime}}, \frac{q_{e} \stackrel{r}{\rightarrow} q_{e}^{\prime} \quad q_{s} \stackrel{m}{\rightarrow} q_{s}^{\prime} q_{n} \stackrel{u}{\rightarrow} q_{n}^{\prime} q_{n} \not f f}{q_{e} q_{s} q_{n} q_{t} \stackrel{r m u}{\longrightarrow} q_{e}^{\prime} q_{s}^{\prime} q_{n}^{\prime} q_{t}}, \\
\stackrel{q_{e} \stackrel{a}{\rightarrow} q_{e}^{\prime} q_{s} \stackrel{m}{\rightarrow} q_{s}^{\prime} q_{n} \stackrel{u}{\rightarrow} q_{n}^{\prime} q_{s} \not h \quad q_{n} \not \supset b \quad q_{n} \not f}{q_{e} q_{s} q_{n} q_{t} \stackrel{a m u}{\longrightarrow} q_{e}^{\prime} q_{s}^{\prime} q_{n}^{\prime} q_{t}} .
\end{gathered}
$$

It is important to observe here that this glue operator ensures the required safety properties independently of the specific implementation of the behaviors of the atomic components (Engine, Sensor, Navigation system and Transmitter), as long as this implementation respects the specified interfaces.

\section{The Glue Expressiveness Hierarchy}

The rule format for defining glue operators that we introduced in Sect.2.2 allows three types of premises: firing, witness, and negative. In this section, we use the notion of glue expressiveness [2] to show that all three types are essential for the expressiveness of the glue that can be defined with these rules. To do so, we compare the following four classes of glue operators:

F: the class of glue operators obtained by using rules with only firing premises (of the form $q \stackrel{a}{\rightarrow} q^{\prime}$ ). Classical composition operators, e.g., parallel composition in CCS or CSP belong to this class;

FW: the class of glue operators obtained by using rules with only positive premises (firing premises, as above, and witness premises of the form $q \uparrow a$ );

FN: the class of glue operators obtained by using rules with only firing and negative (of the form $q \nmid a$ ) premises;

FWN: the universal glue obtained by using rules with all three types of premises.

Let $\mathcal{B}$ be a set of behaviors with a fixed equivalence relation $\mathcal{R} \subseteq \mathcal{B} \times \mathcal{B}$. A glue is a set $G$ of operators on $\mathcal{B}$. We denote by $\mathcal{G}$ lue the set of all glues on $\mathcal{B}$. We denote $G^{(n)} \subseteq G$ the set of all $n$-ary operators in $G$. Thus, $G=\bigcup_{n \geq 1} G^{(n)}$.

To determine whether one glue is more expressive than another, we compare their respective sets of behaviors composable from the same atomic ones. This consists in exhibiting for each operator of one glue an equivalent operator in the other one. We only consider strong expressiveness [2, i.e., where the exhibited glue operator must be applied to the same set of behaviors as the original one.

Although the results we present can also be extended to weak expressiveness [2], where the exhibited glue operator must be applied to the same set of behaviors as the original one with an addition of some fixed set of coordination behaviors, we do not present these extensions to avoid overcharging the paper. 
Definition 6. For a given set $\mathcal{B}$ and an equivalence $\mathcal{R}$ on $\mathcal{B}$, the expressiveness preorder $\preccurlyeq \subseteq \mathcal{G}$ lue $\times \mathcal{G l u e}$ w.r.t. $\mathcal{R}$ is defined by putting, for $G_{1}, G_{2} \in \mathcal{G l u e}$, $G_{1} \preccurlyeq G_{2}$ if, for any $n \geq 1$ and $B_{1}, \ldots, B_{n} \in \mathcal{B}$, holds $\forall g l_{1} \in G_{1}^{(n)} \exists g l_{2} \in G_{2}^{(n)}$ : $g l_{1}\left(B_{1}, \ldots, B_{n}\right) \mathcal{R} g l_{2}\left(B_{1}, \ldots, B_{n}\right)$.

We consider the partial order induced by $\preccurlyeq$, that is we say that $G_{1}$ is less expressive than $G_{2}$ if $G_{1} \preccurlyeq G_{2}$ and $G_{2} \npreceq G_{1}$.

Definition 7. Let $B_{1}=\left(Q_{1}, P_{1}, \rightarrow, \uparrow\right), B_{2}=\left(Q_{2}, P_{2}, \rightarrow, \uparrow\right)$. A binary relation $\sqsubseteq \subseteq Q_{1} \times Q_{2}$ is a simulation iff, for all $q_{1} \sqsubseteq q_{2}$ and $a \subseteq P, q_{1} \uparrow a$ implies $q_{2} \uparrow a$ and $q_{1} \stackrel{a}{\rightarrow} q_{1}^{\prime}$ implies $q_{2} \stackrel{a}{\rightarrow} q_{2}^{\prime}$, for some $q_{2}^{\prime} \in Q_{2}$ such that $q_{1}^{\prime} \sqsubseteq q_{2}^{\prime}$.

We write $B_{1} \sqsubseteq B_{2}$ if there exists a simulation relating each state of $B_{1}$ to some state of $B_{2}$. $匚$ is the simulation preorder on behaviors. The relation $\simeq=$ $\sqsubseteq \cap \sqsubseteq^{-1}$ is the simulation equivalence on behaviors.

Proposition 3. With respect to the simulation equivalence, $F$ is less expressive than $F W$ and $F N$, which are both less expressive than $F W N$.

\section{Glue Constraints, FWN, and BIP}

BIP [17] is a component framework for constructing systems by superposing three layers of modeling: Behavior, Interaction, and Priorities. The lower layer consists of a set of atomic components modeled by transition systems. The second layer models interactions between components. Priorities are used to enforce scheduling policies applied to interactions of the second layer. Below, we provide a succinct formalization of the BIP component model.

Contrary to Def. 2, a behavior in BIP is an LTS (cf. Def. 11). Let $B_{i}=$ $\left(Q_{i}, P_{i}, \rightarrow\right)$, for $i \in[1, n]$, be a set of transition systems and $P=\bigcup_{i=1}^{n} P_{i}$. The composition of $\left\{B_{i}\right\}_{i=1}^{n}$, parameterized by a set of interactions $\gamma \subseteq 2^{P}$, is the transition system $\gamma\left(B_{1}, \ldots, B_{n}\right)=(Q, P, \rightarrow)$, where $Q=\prod_{i=1}^{n} Q_{i}$ and $\rightarrow$ is the minimal relation defined by the following set of rules:

$$
\left\{\frac{\left\{B_{i}: q_{i} \stackrel{a \cap P_{i}}{\longrightarrow} q_{i}^{\prime} \mid i \in I_{a}\right\} \quad\left\{q_{i}=q_{i}^{\prime} \mid i \notin I_{a}\right\}}{\gamma\left(B_{1}, \ldots, B_{n}\right): q_{1} \ldots q_{n} \stackrel{a}{\longrightarrow} q_{1}^{\prime} \ldots q_{n}^{\prime}} \mid a \in \gamma\right\}
$$

where, for $a \in \gamma, I_{a}=\left\{i \in[1, n] \mid a \cap P_{i} \neq \emptyset\right\}$. We call $\gamma$ an interaction model.

Given a transition system $\gamma\left(B_{1}, \ldots, B_{n}\right)$, a priority model $\pi$ is a strict partial order on $2^{P}$. For $a, a^{\prime} \in 2^{P}$, we write $a \prec a^{\prime}$ iff $\left(a, a^{\prime}\right) \in \pi$, meaning that interaction $a$ has less priority than interaction $a^{\prime}$. The system obtained by applying the priority model $\pi$ to $\gamma\left(B_{1}, \ldots, B_{n}\right)$ is the transition system $\pi \gamma\left(B_{1}, \ldots, B_{n}\right)=(Q, P, \rightarrow)$, where $Q$ and $P$ are the same as above, while $\rightarrow$ is the minimal set of transitions satisfying the set of inference rules

$$
\pi \gamma=\left\{\frac{\gamma\left(B_{1}, \ldots, B_{n}\right): q \stackrel{a}{\longrightarrow} q^{\prime} \quad\left\{\left\{a^{\prime}\right\}\left(B_{1}, \ldots, B_{n}\right): q \stackrel{a^{\prime}}{\longrightarrow} \mid a \prec a^{\prime}\right\}}{\pi \gamma\left(B_{1}, \ldots, B_{n}\right): q \stackrel{a}{\longrightarrow} q^{\prime}} \mid a \in \gamma\right\}
$$


Intuitively, the premises of a rule for $a \in \gamma$ mean that, in the state $q \in Q, a$ is possible in $\gamma\left(B_{1}, \ldots, B_{n}\right)$, and, for each interaction $a^{\prime}$ having higher priority than $a$, there is at least one component $B_{i}$ such that the constituent interaction $a^{\prime} \cap P_{i}$ is not possible in $B_{i}$. It is not required that $a^{\prime}$ belong to $\gamma$. In [2], we have shown that the glue of BIP is as expressive as the most general glue defined by inference rules of the form (1).

Example 5. The glue operator for the mutual preemption (Ex. 1) is expressed in BIP by considering the interaction model $\gamma=\left\{b_{i}, f_{i}, b_{i} p_{j}, f_{i} r_{j} \mid i, j=1,2, i \neq j\right\}$ and a priority model given by $b_{i} \prec b_{i} p_{j}$ and $f_{i} \prec f_{i} r_{j}$, for $i, j=1,2$ and $i \neq j$.

Proposition 4. With respect to the simulation equivalence, $F N$ is less expressive than the glue in BIP; FWN and the glue in BIP are not comparable.

An important advantage of BIP is that it allows mastering the complexity of designs by developers: separating glue into two layers corresponding to interactions and priorities drastically simplifies readability of specifications. By redefining the semantics of priority models in terms of the predicate $\uparrow$, it can be shown that this separation can be achieved for the glue operators of Def. 4 .

Separation of the coordination level defined by the glue operators into interactions and priorities allows the application of our previous results presented in [78. By comparing (11) and (3), one can observe that both Interaction layer of BIP and positive glue operators of this paper are uniquely characterized by the interactions appearing in their premises. For the former, interactions are subsets of $2^{P}$; for the latter, they are subsets of $2^{P \cup \dot{P}}$. The sets $P$ and $\dot{P}$ being disjoint, all the results from the two papers cited above can be applied to connectors of the algebra $\mathcal{A C}(P \cup \dot{P})$. This allows considering structured hierarchical connectors instead of exponential sets of interactions (in BIP) or rules (for glue operators of this paper). Connectors are well suited for compositional design and can be manipulated by symbolic techniques [7]. Furthermore, connectors can be synthesized directly from causal rules, which are boolean constraints (implications) on port variables similar to the constraints found in the examples of Sect. 4.

\section{Design Methodology}

Synthesis of reactive systems has been initiated by Pnueli and Rosner. They have shown that synthesis of distributed systems is hard and, sometimes, undecidable 9. Lustig and Vardi have obtained similar hardness results for synthesis, where reusable components must be selected from component libraries [10.

This paper lays ground to a less ambitious, but more tractable design methodology relying on the observation that one of the main difficulties of systems design resides in the concurrent nature of modern software (particularly due to the state space explosion). This methodology can be summarized by the following steps:

1. Choice of the functionalities to be realized by sequential atomic components. This step does not involve specifying coordination among these atomic components. It is usually driven by the functional requirements (e.g., functionalities that must be provided by the system) or target platform hardware 
specifications. Tools and techniques needed for this design step are, in general, well mastered by software engineers.

2. Independent design of sequential atomic components of the system. As mentioned above, development of sequential programs is much less complex than that of concurrent ones and can also be successfully realized by engineers.

3. Specification of state safety properties to be satisfied by the system. The general case complexity of this design step has yet to be investigated. All the properties that we have encountered are obtained as causal rules in a manner similar to that of the example in Sect. 4] (cf. [8]).

4. Automatic glue operator and connector synthesis. This implies that the underlying state safety properties are satisfied by construction.

For safety properties other than state properties, compositional deadlock analysis can be performed by adding (sequential) observer components to the synthesized model.

\section{Related Work}

A number of paradigms for unifying interaction in heterogeneous systems have been studied [12 13 14]. In these works, unification is achieved by reduction to a common low-level semantic model. Coordination mechanisms and their properties are not studied independently of behavior. Our approach is closest to that of [15], where an algebra of connectors is developed that allows one to construct stateless connectors from a number of basic ones.

This paper combines several notions that already exist in the literature. We use boolean constraints expressed in terms of activation and firing variables in order to synthesize glue operators expressed in terms of SOS rules.

In concurrency, the term constraints appears primarily in connection with the constraint automata [1617, where constraints are used to restrict transition non-determinism. These automata are now widely referred to as having guarded transitions. We speak of interaction constraints that characterize mechanisms used in architectures such as connectors, channels, synchronization primitives and result from the composition of actions [18. Architectural mechanisms are used to constrain the interaction among parallel communicating components.

Few authors have considered the approach by constraints in this context, e.g., [19]. The approach used in [20] is close to the one we adopted in our previous paper [8]. The important difference is that, following the separation of concerns principle, we distinguish coordination and data flow among components.

Separating the coordination layer allows to express coordination constraints as boolean formulæ. In [8], we consider causal rules, which are boolean constraints on port variables. Causal rules are used to synthesize connectors to describe interactions among components in BIP. In the present paper, we extend this methodology to the complete Coordination layer (Interactions and Priorities), which requires a more sophisticated notion of boolean constraints on activation 
and firing port variables, where firing variables are exactly the port variables we refer to in 8 .

To the best of our knowledge, few authors in the domain of component-based design use activation or firing variables as we do in this paper, and we are not aware of any work making a combined use of both. The techniques closest to ours can be found in 20, and in the use of clocks in synchronous languages such as Lustre [21], Esterel [22], and Signal [23].

Several methodologies for synthesis of component coordination have been proposed in the literature, e.g., connector synthesis in 24 25 26]. In [24], connectors are synthesized in order to ensure deadlock freedom of systems that follow a given architectural style. The proposed methodology is seriously limited by two factors. Firstly, the proposed architectural style is very restrictive: components can only be connected in a specific way and communicate by passing two types of messages (notifications and requests). Secondly, in order to ensure deadlock freedom, the authors rely on the analysis that requires computing the product automaton representing the complete system, which is impractical for large systems.

In [25], Reo circuits are generated from constraint automata. This approach is limited, in the first place, by the complexity of building the automaton specification of interactions. An attempt to overcome this limitation is made in [26] by generating constraint automata from UML sequence diagrams. A commonly accepted problem of using UML, in general, and sequence diagrams, in particular, is the absence of formal semantics. Although the Reo approach effectively provides such semantics, there is no guarantee that this semantics can be understood by the designer, as the synthesized constraint automata can be rather complex. More importantly, synthesized Reo connectors (with the constraint automaton semantics) have state. Hence, they contribute to the state space explosion hampering verification of the final system properties.

Our approach allows one to directly synthesize stateless glue from safety requirements in a generic setting and circumvents the difficulty of behavior synthesis by applying the separation of concerns principle; it develops the line of [8] and has similarities with the synthesis of circuits from boolean specifications.

Finally, following the common practice, we use SOS rules to define composition (glue) operators. Since their introduction in [4, numerous formats for SOS rules have been proposed and extensively studied in order to provide generic properties of objects defined by SOS rules. The rule formats that we use in this paper represent two very simple special cases of the existing SOS formats. In particular, the format given by (1) is a special case of GSOS [3].

\section{Conclusion}

We proposed and studied a general framework for component-based construction of systems. The framework is based on an SOS-style characterization of composition operators, called glue operators. The presented boolean characterization of glue operators allows moving from heavy SOS-style definition to a lightweight 
boolean representation. The representation distinguishes between firing and activation variables. This distinction is essential for expressing priorities and, in general, situations where the state of one component influences enabledness of an interaction without participating in it. The use of the offer predicate $\uparrow$ is essential for taking into account offers of the composed individual behaviors. This leads to a concept of transition system richer than usual transition systems, which allows to distinguish between composite and atomic behavior.

The equivalence between glue constraints and universal glue can drastically simplify component-based design. We have shown through examples that glue constraints can be used to express given safety requirements. The synthesis of an implementation from such requirements can be automated by computing the firing-full DNF and the corresponding operational semantics rules. This provides means for deriving executable models from declarative safety requirements opening the way for an automated synthesis paradigm similar to hardware synthesis.

Contrary to the algebra presented in [2], the Algebra of Glue Constraints, is very general and intuitive. Indeed, the only axiom different from the standard Boolean ones is, basically, a sanity check: a port cannot participate if it is not active. Any formula in this algebra gives rise to a valid glue operator.

Furthermore, we have shown that, by focusing on the properties (expressed as glue constraints) that do not bear computation, we reduce a very hard and, in general, undecidable problem of synthesizing controllers to a tractable one. The Rescue Robot example shows that such properties are quite natural and can be expressed as glue constraints in a straightforward manner.

Amongst the formalisms discussed in the paper, BIP presents the advantage of being more appropriate for mastering the complexity of designs by developers. The principle of separating glue into two layers corresponding to interactions (glue with positive premises) and priorities (glue with negative premises and a single positive premise) drastically simplifies readability of specifications. The presented results suggest an evolution of BIP semantics to encompass the universal glue defined in this paper.

This work is part of a broader research project around BIP, including the development of the BIP language and associated tool-set for component-based design. The tool-set includes compilers as well as compositional verification tools. It has been successfully applied to modeling and validation of heterogeneous systems [1127]. We will continue our study to further explore relations between three identified approaches: SOS-based, constraint-based, and layered.

\section{References}

1. Basu, A., Bozga, M., Sifakis, J.: Modeling heterogeneous real-time components in BIP. In: $4^{\text {th }}$ IEEE Int. Conf. on Software Engineering and Formal Methods (SEFM 2006), pp. 3-12 (2006) Invited talk

2. Bliudze, S., Sifakis, J.: A notion of glue expressiveness for component-based systems. In: van Breugel, F., Chechik, M. (eds.) CONCUR 2008. LNCS, vol. 5201, pp. 508-522. Springer, Heidelberg (2008) 
3. Bloom, B.: Ready Simulation, Bisimulation, and the Semantics of CCS-Like Languages. PhD thesis, Massachusetts Institute of Technology (1989)

4. Plotkin, G.D.: A structural approach to operational semantics. Technical Report DAIMI FN-19, University of Aarhus (1981)

5. Mousavi, M., Reniers, M.A., Groote, J.F.: SOS formats and meta-theory: 20 years after. Theoretical Computer Science 373(3), 238-272 (2007)

6. Cheng, C.P., Fristoe, T., Lee, E.A.: Applied verification: The Ptolemy approach. Technical Report UCB/EECS-2008-41, University of California at Berkeley (2008)

7. Bliudze, S., Sifakis, J.: The algebra of connectors - Structuring interaction in BIP. In: Proc. of the EMSOFT 2007, pp. 11-20. ACM SigBED, New York (2007)

8. Bliudze, S., Sifakis, J.: Causal semantics for the algebra of connectors. Formal Methods in System Design 36(2), 167-194 (2010)

9. Pnueli, A., Rosner, R.: Distributed reactive systems are hard to synthesize. In: Annual IEEE Symposium on Foundations of Computer Science, vol. 2, pp. 746$757(1990)$

10. Lustig, Y., Vardi, M.Y.: Synthesis from component libraries. In: de Alfaro, L. (ed.) FOSSACS 2009. LNCS, vol. 5504, pp. 395-409. Springer, Heidelberg (2009)

11. Bensalem, S., Bozga, M., Nguyen, T.-H., Sifakis, J.: D-Finder: A tool for compositional deadlock detection and verification. In: Bouajjani, A., Maler, O. (eds.) CAV 2009. LNCS, vol. 5643, pp. 614-619. Springer, Heidelberg (2009)

12. Balarin, F., et al.: Metropolis: An integrated electronic system design environment. IEEE Computer 36(4), 45-52 (2003)

13. Balasubramanian, K., et al.: Developing applications using model-driven design environments. IEEE Computer 39(2), 33-40 (2006)

14. Eker, J., et al.: Taming heterogeneity: The Ptolemy approach. Proc. of the IEEE 91(1), 127-144 (2003)

15. Bruni, R., Lanese, I., Montanari, U.: A basic algebra of stateless connectors. Theor. Comput. Sci. 366(1), 98-120 (2006)

16. Fribourg, L., Peixoto, M.V.: Concurrent constraint automata. In: ILPS 1993: Proc. of the 1993 International Symposium on Logic Programming, vol. 656. MIT Press, Cambridge (1993)

17. Baier, C., et al.: Modeling component connectors in Reo by constraint automata. Sci. Comput. Program. 61(2), 75-113 (2006)

18. Gößler, G., Sifakis, J.: Composition for component-based modeling. Sci. Comput. Program. 55(1-3), 161-183 (2005)

19. Montanari, U., Rossi, F.: Graph rewriting and constraint solving for modelling distributed systems with synchronization (extended abstract). In: Hankin, C., Ciancarini, P. (eds.) COORDINATION 1996. LNCS, vol. 1061, pp. 12-27. Springer, Heidelberg (1996)

20. Clarke, D., et al.: Deconstructing Reo. In: FOCLASA 2008. ENTCS (2008)

21. Halbwachs, N., et al.: The synchronous dataflow programming language LusTRE. Proc. of the IEEE 79, 1305-1320 (1991)

22. Berry, G., Gonthier, G.: The ESTEREL synchronous programming language: Design, semantics implementation. Sci. Comput. Program. 19(2), 87-152 (1992)

23. Benveniste, A., Guernic, P.L., Jacquemot, C.: Synchronous programming with events and relations: the SIGNAL language and its semantics. Sci. Comput. Program. 16(2), 103-149 (1991)

24. Inverardi, P., Scriboni, S.: Connectors synthesis for deadlock-free component-based architectures. In: ASE 2001, pp. 174-181. IEEE Computer Society, Los Alamitos (2001) 
25. Arbab, F., Baier, C., de Boer, F.S., Rutten, J., Sirjani, M.: Synthesis of Reo Circuits for Implementation of Component-Connector Automata Specifications. In: Jacquet, J.-M., Picco, G.P. (eds.) COORDINATION 2005. LNCS, vol. 3454, pp. 236-251. Springer, Heidelberg (2005)

26. Arbab, F., Meng, S.: Synthesis of connectors from scenario-based interaction specifications. In: Chaudron, M.R.V., Ren, X.-M., Reussner, R. (eds.) CBSE 2008. LNCS, vol. 5282, pp. 114-129. Springer, Heidelberg (2008)

27. Basu, A., et al.: Incremental component-based construction and verification of a robotic system. In: ECAI, pp. 631-635 (2008) 